\title{
Philosophiques
}

\section{Une genèse de la vie sociale selon Maurice Blondel}

\section{Léo-Paul Bordeleau}

Volume 2, numéro 1, avril 1975

URI : https://id.erudit.org/iderudit/203022ar

DOI : https://doi.org/10.7202/203022ar

Aller au sommaire du numéro

Éditeur(s)

Société de philosophie du Québec

ISSN

0316-2923 (imprimé)

1492-1391 (numérique)

Découvrir la revue

Citer cet article

Bordeleau, L.-P. (1975). Une genèse de la vie sociale selon Maurice Blondel.

Philosophiques, 2(1), 55-82. https://doi.org/10.7202/203022ar d'utilisation que vous pouvez consulter en ligne.

https://apropos.erudit.org/fr/usagers/politique-dutilisation/ 


\title{
UNE GENÈSE DE LA VIE SOCIALE SELON MAURICE BLONDEL
}

\author{
par Léo-Paul Bordeleau
}

La seconde moitié du XIXe siècle est marquée d'un renouveau de l'esprit scientifique et philosophique. L'espèce de lassitude qu'éprouvent, à cette époque, la plupart des penseurs à l'égard des grands systèmes rationnels déclenche un regain de la recherche sous le signe de la force et du dynamisme.

Ainsi, dans le domaine de la science expérimentale, c'est sous le signe de l'agir que s'exécutent les travaux en laboratoire, que s'acquièrent des notions opératoires, que s'entassent des matériaux précieux, que progresse le savoir. De la même manière, beaucoup d'oeuvres philosophiques, françaises tout au moins, se présentent comme des essais pour guider la réflexion vers une productivité spirituelle; leurs démarches s'inscrivent dans la ligne d'un positivisme spiritualiste dont Ravaisson a bien discerné les signes dans son mémoire de 1867. Ce positivisme spiritualiste, « ayant pour principe générateur la conscience que l'esprit prend en lui-même d'une existence dont il reconnaît que toute autre existence dérive et dépend, et qui n'est autre que son action $»$ '. est essentiellement un effort pour saisir, par la réflexion critique, l'activité spirituelle dans sa production. Cette tendance vers le concret en parturition traduit l'urgence d'un examen de l'action.

Or, de tous les philosophes, Maurice Blondel est le seul à oser, non seulement un examen philosophique de l'action, mais surtout son adoption intégrale comme centre de perspective et de recherche. Au cours de la décade préparatoire à sa thèse de doctorat, ses tentatives de dégager de la pratique et de l'épreuve

1. RAVAISSON, F., La philosophie en France au XIXe siècle, Paris, Hachette, 3e éd., 1889, p. 275. 
concrète de la vie la lumière que l'analyse abstraite ne peut fournir lui enseignent que l'action est le lieu géométrique où convergent les puissances d'en bas et d'en haut. Evidemment, Blondel ne prétend pas tout inventer en proposant l'action comme foyer d'intelligibilité ; sa réflexion est portée par l'esprit de son temps; il reconnaît ses dettes envers de nombreux penseurs dont Aristote, à ce propos, est l'un des plus marquants ${ }^{2}$. Il devra cependant tailler dans du neuf, car, à cette époque, le mot action ne figure dans aucun dictionnaire philosophique ${ }^{3}$. Ainsi, pour Blondel, l'action est ce qui permet de sauver, sans sortir des conditions d'apodicticité imposées par l'esprit positif, la réalité des valeurs spirituelles qui semblent compromises par le positivisme de type comtien.

Par ailleurs, on doit reconnaître que la philosophie blondélienne n'est pas facile à pénétrer parce que son auteur l'articule selon une méthode génétique et un processus d'implications, et parce que toujours il cherche à rendre compte des oppositions et des collaborations entre les forces de la réalité comme entre les théories explicatives, c'est-à-dire à discerner la dualité là où elle est et telle qu'elle est, dans sa raison d'être et son rôle provisoire conduisant à l'unité. Comprendre Blondel c'est ne pas confondre l'ordre de l'exposition et l'ordre de la recherche et savoir qu'il pose sa problématique en fonction du christianisme, celui-ci ne constituant pas tant le postulat que l'hypothèse directrice de son effort philosophique; c'est savoir aussi qu'à ses yeux chaque partie du réel s'intègre avec l'ensemble et vaut par son dynamisme; c'est savoir enfin qu'il a poussé la philosophie jusqu'à montrer que de son insuffisance naît une philosophie de l'insuffisance. Une telle philosophie me paraît dépasser en richesse et en profondeur ces philosophies de l'action de type anglo-saxon qui, dans un parti-pris d'objectivité et d'exactitude scientifiques, réduisent la compréhension de la réalité mouvante à des formules mathématiques ${ }^{4}$.

2. L'Action, t. I, éd., 1949 , pp. 54 à 66 ; pp. 239-240.

3. L'itinéraire philosophique de Maurice Blondel, Aubier, 1966, pp. 34-35.

4. A ce sujet, on peut consulter Agent, Action and Reason, édité par R. Binkley, R. Bronaugh, A. Marras, University of Toronto Press, 1971. 
Ces remarques sont justifiées. Elles veulent faire sentir au lecteur qu'une interprétation et une critique d'un point de doctrine d'un philosophe supposent la mise en place d'un contexte doctrinal et souvent historique. Ce procédé est encore plus souhaitable lorsqu'il s'agit de l'étude d'un thème encore jamais examiné dans son ensemble et pour lui-même. Dans cette perspective, on comprendra mieux l'apparent détour que constitue le déroulement de l'action qui deviendra créatrice de sociabilité. Si l'homme est, suivant les termes de Blondel, une «allergie» (besoin et désir des autres) et une «coénergie» (exercice d'une communion), c'est parce qu'il est d'abord une «synergie» (synthèse d'intention et d'actes).

Mon intention est de présenter le travail de l'action pour arriver à manifester sa dimension sociale. Il sera donc nécessaire de dérouler, dans un premier temps, les étapes antérieures à cette dimension pour ensuite l'examiner en elle-même. Cependant, des limites s'imposent: je néglige l'influence que les milieux intellectuel et social auraient pu exercer sur Blondel ; j'exclus l'étude de la nature proprement dite de la société et la raison d'être de la pluralité des sociétés; je restreins le champ de mon étude à la thèse de 1893, considérée comme le noyau central de la philosophie blondélienne, amorcée dans la thèse complémentaire et mûrie dans la tétralogie ${ }^{5}$. Cette étude n'a donc pas la prétention d'exposer ni d'évailuer la pensée sociale blondélienne en son entier.

\section{I - LE DÉROULEMENT DE L'ACTION}

Dans sa thèse doctorale de 1893, Blondel entreprend l'étude du phénomène de l'action à partir de ses plus élémentaires origines jusqu'à son plus large développement. La difficulté à vaincre est de savoir où se situe la solution positive au problème de la vie ; est-elle de l'ordre naturel des choses, de la science, ou faut-il

5. La thèse doctorale de Maurice Blondel s'intitulait: L'Action. Essai d'une critique de la vie et d'une science de la pratique. L'édition de 1893 fut réimprimée en 1950. La thèse complémentaire étudiait le De vinculo substantiali et de substantia composita apud Leibnitium, publiée en 1893, présentée et traduite par Troisfontaines en 1972. La tétralogie groupe : La Pensée (1934, 2 tomes), L'Être at les êtres (1935), L'Action (2 tomes, 1936-1937), La pbilosophie et l'esprit (2 tomes, 1944-1946). 
recourir à un transcendant? La recherche active de l'homme traverse plusieurs étapes : en reconstruisant et en ratifiant tout ce qui compose le milieu physique, intellectuel, social, sa volonté y trouve à la fois un obstacle et un aliment, mais aussi une insatisfaction. Mais pour Blondel les embarras sont salutaires, l'hétéronomie est un tremplin, la dualité est stimulante et productrice de nouveau; notre pesanteur même est une des composantes de toute notre activité libératrice.

Une première étape, celle de l'analyse des données de la sensation et de la science, dégagera les sources inconscientes de l'action. Une seconde décrira le dynamisme de la vie interne et les sources conscientes de l'action. La troisième étape est celle qui voit l'action se répandre et proliférer au niveau de la vie individuelle. Mais l'action ne se restreint pas dans l'enceinte de la vie individuelle; elle doit, sous les exigences d'un vouloir initial, se répandre à l'extérieur: se multiplier pour s'unir. La quatrième étape, celle de l'action sociale, le justifiera.

Le mouvement de la vie ne s'arrête pas ici : tout esprit aspire à l'infini. Cependant, ces dernières ondes concentriques que sont l'effort humain pour associer le transcendant à son propre agir et les dispositions religieuses de l'action demeureront hors de mon champ d'analyse.

Sans prétendre en reproduire ici le ton et le mouvement, je résume à grands traits les étapes précédant celle qui constituera le centre de ce travail : la dimension sociale de l'action.

\section{Première étape: genèse de la subjectivité.}

Cette étape justifie l'inconsistance de la sensation et de la science face au problème de la destinée humaine, et définit la science du sujet.

La sensation ou la qualité sensible est-elle la donnée la plus immédiate? Non. D'une part, la sensation, qui paraît d'une simplicité absolue, ne saurait se suffire à elle-même puisqu'elle recèle une incohérence interne; elle n'est pas une donnée initiale et univoque puisqu'elle est fondée sur une intégration de faits. En effet, pour être, la sensation doit tout à la fois être sentie, représentée, éprouvée : l'homme n'a de sensation qu'à la 
condition que ce qu'il éprouve soit sien tout en paraissant extérieur à lui-même; la sensation s'énonce par une opposition du sujet et de l'objet. Une sensation est toujours une sensation de quelque chose d'autre qu'elle-même: la conscience de sentir exprime l'impossibilité de se borner à la sensation brute et toute a posteriori. Si la sensation pure est illusion, c'est qu'en fait elle est l'acte commun du corps et de la conscience; on éprouve une nécessité de se représenter le sensible à la fois tel qu'il est senti et autre qu'on ne le sent; on ne peut se représenter le tout d'une chose. D'autre part, et par conséquent, dans la plus simple intuition sensible se cache une dualité qui est à l'origine d'un besoin de savoir. La sensation n'est possible que par un jugement implicite d'objectivation, c'est-à-dire la perception. Il est impossible de placer dans la sensation la mesure du réel ou de l'intelligible puisqu'elle n'en est que le composé le plus élémentaire et le plus instable, et que son rôle se borne à provoquer dans la conscience une "rupture d'équilibre». La sensation «ne commence à être elle-même qu'au moment où l'on cherche, où l'on place en elle autre chose que nous et en nous autre chose qu'elle» (p. 47).

Elle n'est un point de départ qu'à la condition d'orienter notre recherche aussi bien vers ses dessous déterminants que vers les développements ultérieurs.

De l'insuffisance de la sensation naît la science. En effet, l'homme, pour assurer son existence, veut savoir sur quoi compter; il organise les phénomènes en systèmes. En d'autres termes, la curiosité scientifique s'éveille à partir du moment où l'expérience nous avertit «que ce que nous sentons n'est pas la seule, vraie et totale réalité de ce que nous sentons» (p. 48). Cette simple découverte a fait les manchettes de la philosophie et des sciences et a donné lieu à ce que Blondel appelle un «réalisme phénoméniste ». Il étudie le développement de ce courant afin de voir si les sciences positives suffisent à la vie humaine et se suffisent à elles-mêmes. Réussissent-elles là où la sensation échoue? De l'alliance des sciences mathématiques avec les sciences expérimentales résulte le pouvoir d'atteindre la réalité comme par les deux bouts. Les unes semblent régner sur les nombres, les autres sur les phénomènes. Ainsi les sciences positives prétendent 
répondre à tous les besoins humains pratiques et spéculatifs. C'est l'époque du scientisme, celle où les problèmes se concentrent autour de la portée, de la nature et de l'impérialisme de la science, avec Taine, Berthelot, Renan; ce dernier croit que la science est une nouvelle religion, que le savant est le saint des temps nouveaux; d'ailleurs, Fichte n'avait-il pas exprimé ce sacerdoce de la science? Or cette prétention est-elle justifiée? Les sciences positives forment-elles un tout cohérent et suffisant? «Est-ce la science qui explique tout de l'action, ou l'action qui fournit encore à la science de quoi subsister? » (p. 50).

Dans un long chapitre, Blondel montre qu'il y a, entre les sciences mathématiques et expérimentales, à la fois rupture et solidarité, que toutes deux souffrent d'une insuffisance radicale, que leur rapprochement n'est possible que par la médiation de l'action ${ }^{6}$. Il explique en quel sens la médiation de l'action maintient une alliance efficace entre le calcul et l'expérience, entre les sciences mathématiques et les sciences de la nature. La science se fonde sur le postulat de l'action du sujet: "Notre puissance va toujours plus loin que notre science, parce que notre science, issue de notre puissance, a besoin d'elle encore pour y trouver son appui et son terme» (p. 80). En d'autres termes, la science est toujours un fait ou plutôt un acte de la conscience.

Par conséquent, toute vérité scientifique implique un principe d'unité qu'on appelle la subjectivité. Blondel dénonce l'illusion qui fait croire que le savant opère comme s'il était indépendant du monde, comme s'il n'était pas nécessairement un centre de perspective. La subjectivité est le vinculum perceptionis des sciences (p. 90). Cette nouvelle inconnue, réfractaire aux explications des sciences, Blondel la détermine en montrant que l'étude subjective de l'action comporte une rigueur scientifique et qu'elle prolonge nécessairement, en les justifiant, les sciences positives: faire la science positive du sujet, déterminer le lien qui maintient la continuité scientifique de la connaissance mathématique ou expérimentale jusqu'aux faits de conscience.

La conscience émerge du milieu dans lequel elle baigne tout en s'en distinguant; ce milieu est celui des phénomènes. Or

6. L'Action de 1893 , pp. 51-86. 
"le phénomène n'est ce qu'il est, qu'en fonction d'une activité qui contribue à l'engendrer; on ne le perçoit que selon l'ordre même de sa production » (p. 91). Ainsi, la genèse des phénomènes ne se comprend qu'à partir de la conscience, alors même que «le sujet n'est pas dans la série» (p. 92). Cette présence nécessaire et féconde qu'est la subjectivité n'est pas la simple série des phénomènes conscients; elle est ce qui fait que le phénomène peut s'intérioriser et devenir représentatif de l'univers. En effet, dans les sciences exactes comme dans l'analyse expérimentale, le phénomène est d'autant mieux perçu et objectif qu'il révèle une subjectivité croissante. Il y a donc une interaction réciproque de l'intériorité et de l'extériorité. Cette force synergique forme des centres d'équilibre, «des groupements plus ou moins stables» (p. 93). La vie est «l'organisation d'un petit monde qui reflète le grand $\gg(p .93)$; elle apparaît avec l'individualité. Le vivant est «comme un système concentré de forces coordonnées» (p. 94). De même que la science est née de l'insuffisance de la sensation, la conscience apparaît du fait que l'instinct ne suffit pas à résoudre les problèmes que pose la vie d'un être doué de raison.

La conscience est donc un dynamisme que seule une science spécifique peut expliquer. Ce dedans, d'où procède le positif, échappe à la connaissance positive. C'est par l'analyse de la constitution et de la formation du phénomène qu'on pourra saisir en acte le progrès même de la vie subjective; on verra alors surgir des sciences positives, parce qu'il y était, le fait psychologique. Le phénomène n'existe que s'il $\mathrm{y}$ a action du sujet. Le subjectif est de l'objectif concentré. Or, seule une science de l'action peut cerner la conscience comme telle. «La véritable science du sujet c'est celle qui, considérant dès le point de départ l'acte de conscience comme acte, en découvre par un progrès continu l'inévitable expansion» (p. 100). La conscience draîne et concentre toutes les forces de la nature; l'acte de conscience est une réalité positive.

Par conséquent, les sciences positives établies sont incompétentes à résoudre le problème de la destinée humaine parce qu'elles se rabattent sur le mécanisme externe des phénomènes. Une science du sujet s'impose, toute aussi rigoureuse et positive 
que les précédentes grâce à la médiation de l'action. Il s'agit bien d'une science de par son caractère d'universalité et même de par la possibilité de relations ou de corrélations répétables : «il ne faut pas que mes raisons, si elles sont scientifiques, aient plus de valeur pour moi que pour autrui » (p. XVII). La route est maintenant déblayée pour l'étude de la conscience proprement dite.

\section{Deuxième étape: genèse de la réflexion et de la liberté.}

Cette seconde étape décrit le dynamisme de la vie interne et les sources conscientes de l'action. La naissance proprement dite de la conscience, ou l'éveil de la pensée, nous est inconnue : «Impénétrable demeure la naissance de ce qu'il y a de plus admirable et de moins admiré, la lumière intérieure » (p. 105). Blondel ne cherchera qu'à retracer sa genèse.

La conscience est d'abord un monde clos qui s'épanouit doublement: elle s'ouvre sous les poussées inconscientes de la vie pour ensuite s'élargir aux dimensions de l'univers. Le réel est travaillé par une dynamogénèse. Des synthèses se constituent: la vie, l'instinct. Mais les forces de la nature sont aussi assumées par l'homme sous des formes de synthèses mentales qu'on appelle motifs. Le motif est le résumé, «le député d'une foule de tendances élémentaires qui l'appuient et le poussent; il résulte de causes plus lointaines et plus générales; il est la conclusion de tout un système antérieur ... » (p. 106). Il oriente le dynamisme des mobiles. Il devient lui-même mobile. «On ne pense (c'est dans l'ordre) qu'après avoir agi, et en agissant, et pour agir » (p. 109). De plus, le motif n'est jamais une idée unique: la conception d'une action implique qu'on envisage la possibilité d'autres actions. Dans la synthèse mentale spontanée, la conscience naît en vertu d'une loi de contraste; mais ce contraste ne s'explique et ne se comprend que par rapport à ce pouvoir d'inhibition qu'est la réflexion. «Ce rapport, dans la conscience, des parties contrastantes entre elles et avec le tout, c'est à proprement parler la réflexion» (p. 112). La raison apparaîtra comme le pouvoir d'unifier des idées complémentaires; car «cette pluralité des états solidaires et opposés n'est possible que par l'action immanente d'une puissance capable d'embrasser toute la multi- 
plicité des contraires dans une unité supérieure, et qu'il faut bien appeler la raison» (p. 112).

Or, aux yeux de Blondel, raison et liberté sont tellement impliquées l'une par l'autre qu'il ne croit pas pouvoir traiter l'une avant l'autre sans fausser les perspectives? La liberté, c'est la raison libérante. Cette raison libérante a partie liée avec le déterminisme: "Nécessairement produite à la conscience, la liberté s'exerce nécessairement» (p. 124). Qu'est cette liberté nécessitée et nécessitante, dont la genèse est aussi impérative que son exercice?

D'une part, déterminisme et liberté s'appellent et s'entretiennent: «La liberté, loin d'exclure le déterminisme, en sort et en use ; le déterminisme, loin d'exclure la liberté, la prépare et la produit » (p. 120). La liberté est comme un point culminant servant de partage entre le déterminisme de la nature et la détermination personnelle. Il ne dépend pas de l'homme d'être libre ou non; être libre, ce n'est pas seulement vouloir un acte déterminé, c'est donner à ce vouloir l'infini comme puissance et raison: «la conscience de l'action implique la notion d'infini; et cette notion d'infini explique la conscience de l'action libre» (p. 118).

L'idée d'infini et celle de liberté sont donc liées comme deux modalités de la conscience s'impliquant et s'engendrant l'une l'autre. L'homme n'a conscience d'agir que lorsque son action porte une marque originale; ce qui n'est pas échapper au déterminisme, car, celui-ci, en action, a abouti nécessairement à la conscience de la liberté qui en est devenue l'âme. En effet, la liberté, en prenant conscience d'elle-même, plutôt que de refouler le déterminisme entier de la nature, l'utilise et le ratifie: elle veut ce qui permet de vouloir. Certes, la volonté de l'homme «n'a pas besoin de savoir tout ce qu'elle veut pour vouloir effectivement tout ce qu'elle est 》 (p. 41, note 1); elle ne le pourrait pas d'emblée; c'est pourquoi elle endosse le tout de ce qui la précède, quitte à tâcher de lever cette ambiguïté intimement lice à la vie. En d'autres termes, l'homme échappe à la pression déterminante des déterminismes antécédents parce qu'il les assume d'abord, et aussi parce qu'il est puissant de l'idée 
d'un infini déjà impliqué par toute synthèse de motifs: tout acte libre implique une transcendance par rapport au déterminisme. L'action «n'est consciente de sa propre initiative qu'en s'attribuant un caractère d'infinitude et de transcendance» ( $p$. 119).

D'autre part, la liberté s'exerce nécessairement. « Ne pas vouloir vouloir, c'est donc toujours vouloir» (p. 125). Mais si la liberté est nécessairement produite et agissante, n'est-elle pas un simple automatisme spirituel... ou si elle est seule capable de résoudre l'acte, n'est-elle pas un pouvoir purement arbitraire? «Fatalement produite, elle ne se conserve que librement» (p. 129). Comment justifier ce paradoxe? La liberté s'incarne nécessairement dans des motifs particuliers qui en plus sont contrastants; se soumettant à une hétéronomie, elle confère à l'objet de ses préférences un surcroît de valeur. «A ce qui est insuffisant pour la déterminer, elle ajoute sa propre suffisance pour se déterminer» (p. 130). Il y a une logique de la liberté qui a son principe dans le vouloir même dont le but est d'égaler son acte effectif à son aspiration intime.

Ainsi, la liberté est la vraie raison et la vraie fin de l'action; mais elle ne peut se prendre elle-même pour raison et pour fin qu'en se connaissant; en se connaissant, elle «se pose devant elle comme objet, comme but, comme fin particulière, au-dessus des autres motifs en droit, parmi les autres motifs en fait» (p. 131-132). Mais en se révélant à elle-même, en se proposant comme fin, elle découvre ses limites. «Par un singulier effet d'optique intérieure, elle qui, dans le premier élan de la conscience, est si pleine d'elle-même et si séduisante, paraît vide et inerte quand il faut en faire un but pour la volonté » (p. 132). De nouveau est ressentie la disproportion entre la volonté voulante et la volonté voulue. D'où l'idée d'un dépassement constant, car le vouloir fondamental est orienté vers ce qui a un caractère de bien et de fin. Or il n'y a pas de volonté efficace sans discipline, ni discipline sans renoncement.

Le devoir est donc indispensable à la liberté. Une autonomie immédiate est impossible; l'appel du devoir ne laisse pas de repos; l'homme a moins à vouloir ce qu'il est qu'à devenir ce qu'il veut. Il faut que, pour ne pas s'emprisonner en lui-même 
après avoir absorbé le dynamisme de la nature, le sujet se soumette à une loi d'abnégation, que pour être soi il se contraigne, il se cherche et se réalise ailleurs qu'en lui. La volonté est avide de s'étendre; la liberté ne se conserve et ne s'épanouit qu'en se dépassant elle-même; la liberté est le devoir qu'a le moi de s'accomplir en s'actualisant. Ainsi, la solution au problème de la vie n'est pas seulement sensible, scientifique, psychologique; elle est morale. L'homme a pris conscience de sa liberté en agissant; elle s'est révélée comme un motif, puis comme une fin, mais une fin intermédiaire; elle ne se conserve qu'en s'objectivant. «Ainsi née de l'action, la conscience de la liberté et de l'obligation morake tend à l'action» (p. 139).

\section{Troisième étape: genèse de la vie individuelle.}

Jusqu'à présent, on a assisté au déroulement des conditions qu'impliquent la conception, la croissance interne et la production de l'action. Entre le déterminisme antécédent à la liberté et le déterminisme conséquent, il y a une continuité quoiqu'une transformation s'est produite: la nécessité subie est devenue une nécessité voulue. Avec la troisième étape, on assiste à l'édification du moi ; c'est la genèse de la vie individuelle; on y voit vivre, se répandre et proliférer l'action dans ce premier champ qu'est l'organisme.

L'action est véritablement constitutive de la vie individuelle; elle unit en faisceau les forces éparses de l'organisme; elle sert de médiatrice entre les formes de l'activité corporelle et celles de l'activité spirituelle; elle introduit l'unité dans l'être de l'homme; elle est le ciment de la vie organique et le lien de la conscience individuelle. Il faudrait ici analyser cette synergie de l'action : comment le corps apparaît dans la conscience, comment cette résistance rentre dans le développement du vouloir, comment la passivité, la passion et le travail organique dérivent de causes subjectives. En somme, il s'agirait de justifier l'intégration de toutes les forces actives et passives de la vie corporelle nécessaires à la recherche de la destinée humaine, et de voir comment à ce niveau la volonté initiale ne laisse rien pour inutile. Je n'en donne qu'un aperçu. 
La volonté doit peiner pour arriver à ses fins : c'est la voie unique de son progrès. Le caractère pénible de l'effort tient à des obstacles psychologiques et à des complications morales. Blondel reprend ici le vieux débat biranien sur l'effort. Cependant il faut éviter de comprendre le psychologique et le moral à partir du physiologique; au contraire, c'est celui-ci qui s'éclaire à la lumière de ceux-là. Blondel montre que l'effort organique est un complexe de forces, les unes afférentes ou extérieures à l'homme, les autres efférentes ou intérieures à l'homme. Il y a une première action afférente de l'organisme qui permet de saisir les origines organiques et inconscientes de la conscience. "L'origine physiologique de la conscience est inconsciente» (p. 151). Ce seuil franchi, «il y a une première action efférente de la pensée qui est perçue seulement dans son essence subjective» (p. 151); c'est une initiative du réfléchi par rapport à l'organisme. Puis «il y a une action efférente de la volonté qui demeure inaperçue dans ses effets corporels » (p. 152), mais qui deviendra visible dans ses mêmes effets organiques. «Ici est le point décisif, le point où le corps apparaît à la conscience et où la passivité entre dans l'action même, le point où du conflit intérieur des tendances surgit le sentiment de l'organisme, le point enfin où s'opère la transcription du spirituel en corporel » (p. 153).

Le corps est à la fois un obstacle et un moyen de réaliser l'homme. Son action prend corps par l'action du corps. «Et ce qu'il faut entendre par le corps de l'action c'est tout ce qui, en nous et hors de nous, nous sépare de nous-mêmes» (p. 154). De plus, «il y a une action efférente de la volonté qui, imaginée sous une forme organique, est inaperçue dans ses effets organiques pour n'être perçue qu'en une représentation motrice » (p. 154). Enfin, une action afférente de l'organisme constitue la réponse pratique à laquelle la volonté s'attend. Ainsi entre la première et la dernière action afférente de l'organisme, le corps, de mien, devient moi ; il est assumé. L'action du corps alimente la vie consciente et celle-ci dirige l'action du corps. «Il y a donc un perpétuel circuit» (p. 159). Ce mouvement circulaire construit à la fois la personnalité humaine et l'épuise, d'où l'aspect laborieux du travail. Et le fait que l'homme ne réalise jamais tout ce qu'il projette engendre un sentiment de déception. "Car vouloir 
et faire sont deux» (p. 162). En effet, le plus laborieux est d'accomplir ce que la volonté indique de faire.

Mais l'action est le ciment de l'individualité consciente; évoluant selon une progression cycloïdale, elle emporte les tendances opposées pour en faire un tout cohérent; elle est synthétique ou synthétisante «Il n'y a d'unité, en notre organisme complexe, que par cohésion, et de cohésion que par coopération » (p. 182). Il n'y a donc qu'un moyen de contenir, de régler et d'utiliser les forces diffuses en l'homme, c'est de les capter.

Toutefois, si l'action parvient à obtenir, chez l'individu, une homogénéité intérieure, elle révèle, ce faisant, l'insuffisance de la vie individuelle: l'action «n'est pas l'expression intégrale et définitive de la vie unie, employée, concertée dans son entier » (p. 198). Il y a une disproportion des conditions élémentaires et des exigences de l'activité voulante et voulue qui fait naître l'idée de la finalité, qui explique la cause finale; on ne va de l'avant que si l'on n'est pas en sécurité ni en suffisance. La volonté originelle de l'homme le projette toujours plus loin, et ceci dans le but de s'égaler à elle-même. La dimension altruiste apparaìt chez l'individu lorsque, sous la force attractive de la fin, il se projette hors de lui. La personne ne naît de l'individu qu'en s'assignant une fin impersonnelle. «L'homme n'est homme que par ce qu'il a en lui de vie universelle» (p. 183). L'homme doit agir pour, avec et par les autres. La conscience individuelle, consciemment ou non, est une conscience universelle. Pour réussir à être mieux et plus complètement un, on ne doit, on ne peut rester seul. La personnalisation n'acquiert sa force que par et dans la socialisation.

Il y a donc en l'homme - c'est un besoin de l'expansion volontaire - un sentiment obscur et permanent de toutes les vies étrangères à la sienne. L'exemple de l'enfant est révélateur : ne vivant que pour soi, il n'est pas en soi ; il deviendra personne le jour où il saura prêter un moi à autrui. Ainsi la vie d'un homme c'est le concours de tout le reste; agir, c'est se confier à l'univers, organiser un monde conforme à son voeu. Or ceci ne peut se réaliser que par le passage de l'action individuelle à l'action sociale. 
Il était nécessaire de montrer, sommairement, comment l'action construit la subjectivité, comment sa dialectique ascendante fait apparaître que chaque synthèse porte en elle sa limite et sa contradiction, comment elle appelle l'intersubjectivité. Il reste à préciser, dans une seconde section, le passage de l'action individuelle à l'action sociale.

\section{II - LA DIMENSION SOCIALE DE L'ACTION}

Cette quatrième étape nous fera suivre le progrès de l'action depuis l'enceinte de l'individu jusqu'au point où la volonté initiale qui anime toujours ce mouvement d'expansion attend et réclame l'intime concours d'autrui. Comment l'action, plantée dans l'organisme qu'elle a unifié, se produira-t-elle au dehors? Comment réussira-t-elle à devenir fonction sociale, à créer une société ? En somme, il s'agit de rendre compte de la genèse de la personne sociale et de la vie sociale, de vérifier comment, selon les termes mêmes de Blondel, synergie est à la fois exergie et allergie.

\section{L'exergie}

La vie individuelle a une expansion inévitable; c'est de cette apparente nécessité dont il faut rendre compte. Que signifie cette sortie, cette exergie? Comment justifier l'expansion immédiate et l'expression d'abord sensible de l'action?

Un fait demeure: l'homme marque le milieu où il vit. Il est comme un appareil vibrant qui réagit au moindre choc. Mais à la différence d'une machine ou d'un corps brut, il enregistre pour transformer ad modum recipientis qui, dans ce cas, est humain; ses réponses aux stimuli sont modifiées, organisées. Non seulement l'homme agit comme tous les corps bruts, mais son influence, son action déterminée s'exprime d'une manière déterminée; l'homme n'est pas dans le monde à la façon d'un objet dans une boite. "L'action a son expression, et pour ainsi dire sa trace ou sa physionomie propre» (p. 205). Dans toutes les manifestations, c'est le moi actuel qui s'exprime dans sa totalité ; "quand l'intention, pénétrant dans les membres, a remué les organes, c'en est fait ; ce qui paraissait être en dehors de l'enchaînement des forces physiques est rentré dans l'engre- 
nage» (p. 205). On soupçonne déjà l'enracinement cosmique de la personne et de la société.

Au niveau de cette première expansion, de quel moyen l'action dispose-t-elle pour se lier avec le monde extérieur? Pour que l'action humaine modifie plus ou moins l'univers, elle doit s'exprimer forcément par un signe; elle est même un organisme de signes (p. 204). Le signe devient un moyen privilégié de communication. Mais si le signe sensible est une suite naturelle de l'opération intérieure à l'agent, ce n'est pourtant pas ce côté matériel qu'il faut scruter; cet aspect relève de l'étude des phénomènes propres aux sciences positives. Ce qu'il faut expliquer, c'est le sens de cette expansion naturelle, c'est l'inspiration, le rôle, le but des signes qui font de l'individu un centre de rayonnement, c'est le sens subjectif auquel l'action doit sa physionomie singulière. "Quelle est la raison profonde de tout son déploiement dans le monde extérieur?» (p. 206). Quelle est la nature et la portée du signe inhérent à toute opération de l'homme?

L'action est plus qu'un fait ou un phénomène; elle est chargée de subjectivité ; elle garde, en se produisant au dehors, le caractère cohérent et ordonné de la synthèse individuelle dont elle est le prolongement; elle ne perd pas sa fonction synergique. C'est encore toute riche de ses antécédents qu'elle est un signe dans le monde où elle apparaît.

Qu'y a-t-il de si unique dans un signe? Qu'est-ce qui fait qu'un geste, par exemple, esquisse souvent tout un drame en action? C'est le fait que tout signe sensible traduit la présence et l'action singulière de l'individu; il est un système intelligible de phénomènes dans lequel s'exprime l'intention subjective. Ainsi tout acte en l'homme constitue, hors de lui, un réseau ténu et fragile, mais organisé et expressif, comme un système complexe de mouvements. Et justement, ce qui constitue l'originalité de chaque acte dans le fait qui le manifeste, c'est «l'unité des relations intelligibles qui forment la synthèse ou l'organisme même du signe» (p. 208). Le signe dépasse la simple opération organique; il y a en lui un commerce de l'agent avec autre chose que l'agent; il est riche de ses antécédents et de quelque chose de plus. 
Non seulement le signe exprime l'intention subjective, mais aussi il achemine l'intention primitive vers son achèvement; il est le lien entre l'expression naturelle de l'acte et la fin intentionnelle de cet acte. C'est pourquoi l'action s'exprime forcément. C'est d'ailleurs le désir même de la volonté. Cette expansion nécessaire a donc un sens que la volonté en quête de son accroissement appelait de toute son énergie; tout sert. C'est ainsi que la volonté commence à pénétrer le monde de ses intentions comme elle avait déjà pénétré l'organisme. «Elle prétend gagner l'univers et le dominer en l'absorbant» (p. 209). Le signe qui exprime au dehors l'opération motrice devient une sorte d'invasion conquérante et une absorption de l'univers par la volonté. Cette invasion conquérante du monde extérieur est une autre façon de la volonté de se concentrer et de recueillir toujours davantage.

Cependant, il n'est pas facile d'assimiler l'univers et de le dominer. Impuissante à se rendre d'emblée immanente à l'univers, l'action est forcée de l'appréhender avec la forme restreinte et condensée d'un objet déterminé. C'est là que le signe, par sa capacité de fixer la tendance fondamentale sur un objet partiel, joue son vrai rôle. "Le signe primitif de l'opération intime, en projetant devant nous notre dessein spontané, le propose à la connaissance distincte; il en fait pour la réflexion un objet partiel» (p. 210). Ce n'est plus l'idée de finalité mais la fin elle-même que la volonté opérante continue à poursuivre. L'expression sensible de l'acte indique donc une tendance du vouloir vers un but ultérieur. En effet, le signe, de par son essence même, interpelle les sujets du milieu dans lequel il apparaît; il est modifié et enrichi par cette interpellation. En d'autres termes, pour être authentique, le signe doit signifier quelque chose qui puisse être capté par autrui; comme toute parole parlée est une parole entendue, le signe exprimé est un signe interprété. Poser le signe, c'est poser le signifié en même temps que le signifiant et le destinataire. C'est la raison pour laquelle l'action semble être dans un perpétuel devenir; dès qu'une fin paraît atteinte, elle devient un moyen, un échelon pour aller au delà.

Par conséquent, si l'individu, en agissant, sort de lui-même, ce n'est pas uniquement à cause d'un superflu ou d'une surabon- 
dance, mais aussi parce qu'il y a en lui insuffisance, pénurie, dénivellement entre l'initiative et les résultats de son opération interne. On retrouve la dialectique de l'action, de la volonté voulante et de la volonté voulue. Entre ce que la vie individuelle est et ce que l'individu veut, il y a un déséquilibre. Fin interne s'allie à fin externe, qui à son tour s'intériorise pour s'allier à une autre fin externe et ainsi jusqu'à ce que la volonté trouve sa plénitude. En d'autres termes, pour retrouver l'accord perpétuellement rompu entre le réalisé et ce qui est voulu, l'homme doit se faire la cause efficiente d'une cause finale et l'attirer à lui pour devenir lui-même cause finale.

Donc, toute action intéresse nécessairement le milieu étranger où elle s'exprime. Cette expression n'est possible que par une complaisance immédiate d'autre chose que l'agent. "L'action ne se produit dans l'atmosphère dont elle est enveloppée qu'en intéressant à son apparition même ce qu'elle ignore et ce qu'elle n'atteint pas encore » (p. 212). La collaboration, le concours actif d'alliés étrangers et en apparence indépendants reste compris dans le sens originel du signe. «Nous sommes forcés de donner, parce que forcément nous avons à recevoir » (p. 201). Mais au fur et à mesure des requêtes progressives de la volonté, augmentent aussi les difficultés et les efforts pour les résoudre. Un monde de consciences ne se plie pas à volonté. Comment la volonté obtiendra-t-elle une active coaction? Une certaine domination peut s'exercer par les enseignements de l'expérience et des sciences. Mais c'est justement de ce succès qu'il faut rendre compte. A quelle condition cette réussite est-elle possible? Que suppose-telle dans l'agent qui la provoque et dans l'agi qui la permet? Il importe maintenant d'analyser la réponse provoquée par l'agent, ce qui fait suite à l'exergie de l'action, c'est-à-dire l'allergie ou l'action d'autrui.

\section{L'allergie}

L'action a fait éclater le cercle de la subjectivité individuelle. Grâce à la médiation du signe, tout acte fait appel à une sorte de complaisance et de collaboration extérieures: un geste et une parole ne sont signifiants que par le milieu où ils se manifestent. «Tout acte vient de l'agent, mais pour aller directement à l'agi, 
sans lequel il n'est pas » (p. 214). Le milieu est donc nécessaire à la façon humaine d'être et d'agir. Chacun des actes de l'homme est modifié par l'opinion qu'il a des opinions qu'ont de lui les autres personnes. Bref, son action le dépasse; elle est plus que sienne, étant une obtention de secours. Comment expliquer cette requête nécessaire du concours d'autrui?

De nouveau s'exerce la dialectique de la cause efficiente et de la cause finale. «L'idée d'une fin déterminée à poursuivre suppose déjà l'expression immédiate de l'intention idéale dans le signe réel qui en est l'oeuvre première» (p. 215). Dans l'orientation de son activité, l'homme a toujours à tenir compte du terme de son déploiement: pour sa volonté, la cause finale doit devenir cause efficiente, même si celle-ci ne produit quelque chose que parce qu'elle est corrélative à une finalité. "La cause finale est, en partie, sa cause efficiente » (p. 215). C'est-à-dire qu'en pratique on veut plus que la fin prévue et prédéterminée: on veut aussi son effet. Ainsi, la volonté, se proposant de s'adapter tout, se prolonge dans l'action pour naître au monde dans le but de se l'assimiler. Il y a donc une coalition nécessaire à la production d'une oeuvre. L'acte devient la synthèse de deux puissances concourantes; il suppose la convergence de deux séries de phénomènes, l'une partant de l'agent et l'autre suscitée ailleurs. "Toute production exige le concours de deux acteurs $\gg$ (p. 225). Le concours étranger, l'allergie, se place comme intermédiaire entre ce que la volonté est déjà et ce qu'elle veut être.

A ce second niveau d'expansion, celui du concours d'autrui préparé par le mouvement des forces cosmiques et physiologiques, le signe continue à être un moyen privilégié de communication. Mais le signe a dépassé la simple opération organique; il se présente comme une oeuvre. Blondel le considère comme un sujet secondaire, conme une intention qui a pris corps et vie, comme un être détaché du sujet agissant: "C'est un concours étranger a parte acti, qui permet la plus rudimentaire expansion a parte agentis $\gg$ (p. 208). Ce concours, ou ce besoin de collaboration, était déjà inscrit dans le signe. En effet, le signe qui avait permis l'exode de l'action et qui est entaché d'une tendance vers une fin ultérieurement voulue, n'opère que s'il intéresse à sa production quelque chose d'étranger à la vie individuelle; il est 
médiateur. Par lui, l'action personnelle exige l'action d'autre chose pour se parfaire. Mais par ce fait même elle se trouve modifiée et échappe, en partie, au contrôle de son auteur; les déceptions causées par la portée imprévue d'un geste ou d'une parole le montrent bien: "Tantôt plus, tantôt moins, et jamais juste ce que nous voulions» (p. 217). Pour essayer de remédier à cette situation d'insuffisance, l'homme cherche le concours d'autrui comme une sorte de sécurité et d'approbation: le « je», pour se trouver, est contraint de sortir de lui-même et de former un «nous». La naissance d'une coaction s'impose, c'est-à-dire une certaine contrainte jointe à une convergence et à une union d'activité.

Si l'homme n'agit jamais seul, de quelle manière son action provoquera-t-elle et même contraindra-t-elle l'action d'autrui ? Il est nécessaire de revenir sur le rôle que doit jouer le signe. Blondel nous propose de dépasser le phénomène sensible produit par l'agent pour s'attacher à ce qui rend sensible le phénomène: «voir encore comment la production victorieuse de l'acte n'est possible que par ce qu'il y a de subjectif hors de l'agent même : (p. 218).

Pour pénétrer l'univers extérieur, pour intéresser les autres, l'action doit s'aliéner, se faire objet d'autrui, devenir l'aliment du milieu, la source et le stimulant d'autres énergies: "Nous rentrons dans le déterminisme de la nature pour le solliciter à notre gré et l'orienter vers nos fins. Nous le sollicitons comme il nous sollicite » (p. 218). Porteuse d'une intention, et grâce à la médiation du signe qui lui conserve sa physionomie individuelle, l'action devient une force subjective capable d'organiser «d'autres spontanéités et de les faire servir à ses desseins » (p. 218). En d'autres termes, une confrontation se produit, par le véhicule du phénomène sensible, entre la cause efficiente et le terme de déploiement de la volonté; ce terme fournit les moyens, et, avec plus ou moins de résistance, devient à son tour une cause efficiente. » Toute oeuvre faite suppose deux causes efficientes qui se correspondent et se complètent : l'une et l'autre sont mutuellement la condition nécessaire de leur succès commun. Et l'âme de cette coaction, c'est une intention initiale» (p. 219). 
En somme, on ne domine les phénomènes qu'en les utilisant comme signes et en se donnant à eux comme signes. Le rôle de la volonté, c'est moins d'agir comme un dictateur que de susciter et d'orienter les puissances extérieures, de mettre en présence des forces naturelles. Ainsi pour agir et réussir en quoi que ce soit, il faut savoir s'y prendre. Mais l'efficacité d'une telle coaction est due à une subjectivité qui s'est extériorisée: «Partout où il y a ébauche d'une vie subjective, c'est-à-dire partout, il est donc possible d'opérer » (p. 221). L'homme n'agit donc pas directement sur les faits positifs, mais, à travers les signes qu'ils sont, il atteint les forces obscures capables de les produire : «Agir, c'est pénétrer par tact et par divination dans l'intimité fermée d'autres sujets et les intéresser à soi » (p. 222).

Cette nécessité du subjectif, Blondel n'en fait pas une étude métaphysique; d'ailleurs, à cette époque et peut-être jusqu'en 1937 où il publie le deuxième tome de la seconde Action, Blondel fait une phénoménologie qu'il présente comme suffisante à ellemême ${ }^{7}$. Pour le moment, il juge qu'on n'a pas besoin de recouri: à la métaphysique pour établir la vérité du dynamisme externe qu'implique la continuité mécanique des faits déterminés et solidaires : «Qu'il y ait des sujets étrangers à l'agent, c'est un phénomène de même ordre que l'existence du sujet lui-même » (p. 225).

L'action s'est produite à l'extérieur et a subi une transformation qui est dans la ligne même du vouloir. «L'agent se met dans ce qu'il fait ; et ce qu'il fait le façonne » (p. 223). L'action s'édifie en oeuvre. C'est désormais de ce point de vue qu'il importe de se placer.

L'oeuvre devient un centre d'équilibre; autour d'elle s'instaureront une collaboration et une intimité entre ceux qui coopètent; elle devient un moyen pour communiquer avec autrui; elle est synthèse effective. «L'intention idéale semble tout entière tirée de l'initiateur; la réponse paraît venir tout entière du collaborateur ; mais, en fait, il y a réciprocité de la forme et de la matière, et dans l'oeuvre il y a double opération symétrique : chacun croit tout faire 》 (p. 223). Etant à la fois le produit de

7. On croit remarquer un changement de perspective dans L'Action. Tome 2 : l'action humaine et les conditions de son aboutissement. Paris, P.U.F., 1937, p. 187. 
tel homme et celui d'un concours étranger, l'oeuvre échappe aux deux ; elle déborde le milieu de ses co-auteurs, elle a une portée plus large; au-delà de toute fin particulière se trouve une fin plus générale. Que signifie cette nécessité de dépassement? Comment l'action exercera-t-elle une influence sur des agents différents de son auteur initial tout en respectant leur indépendance? Si c'est encore le jeu d'une secrète ambition du vouloir, en quoi consiste cette ambition?

Se situant au point d'interférences de plusieurs causes efficientes, l'oeuvre achevée, non seulement se pose en spectacle, devient un objet «où nous apprenons à nous voir » (p. 227), mais elle est aussi faite pour être vue : «Ce qu'on fait, on le fait encore pour autre chose qu'on ne croit» (p. 228). L'action se détache; elle acquiert une certaine indépendance et une certaine vie impersonnelle: "Notre idée, en même temps qu'elle est nôtre, est une idée; et dans toute idée il y a un principe universel» (p. 228). L'homme veut mettre dans son acte ce qu'il y a d'universel en tout sujet: la conscience individuelle est une conscience universelle. L'homme n'agit jamais pour lui seul ni par lui seul.

Pour illustrer cette tendance, Blondel prend l'exemple de l'oeuvre d'art. Ailleurs il écrit : c'est elle qui «nous fraie une des avenues les plus pénétrantes d'introduction à l'être : c'est elle qui, concourant à une «science du singulier» et au progrès, à la sauvegarde de la pensée concrète, épouse et féconde la métaphysique véritable, au lieu d'être asservie à une idéologie ${ }^{8}$.

L'oeuvre belle a une certaine suffisance absolue; non seulement elle déborde la vie individuelle où elle avait pris racine, mais elle dépasse le monde du spectateur; elle acquiert une indépendance relative ; elle est comme affranchie du temps et de l'espace; plutôt, elle devient omnitemporelle. Elle est signe de l'universel; du moins elle est signe d'une réalité plus grande qu'elle. Reprenant une idée kantienne, Blondel pense que dans toute action vraiment humaine, celle qui se présente comme une oeuvre, il y a une finalité sans fin. L'oeuvre est prolifique et source d'influence! «On ne peut, on ne veut pas enfermer sa

8. Itinéraire philosophique, Paris, Aubier, 1966, pp. 74-75. 
vie en soi : en agissant, on agit comme pour tous et en tous 》 (p. 230). Ainsi, l'homme se dupe s'il croit être sa propre fin. Il y a chez lui un désir, plus, un besoin de faire de l'acte une sorte de création distincte de son auteur, de lui donner une portée universelle. C'est d'ailleurs dans la logique même de l'action: de l'intention à l'influence il y a une suite logique: "Toute oeuvre produite est une propagande en acte» (p. 231). Il serait donc naï de penser qu'on peut agir comme si on était seul ; la pensée comme l'action de chacun sont lourdes de conséquences et de responsabilités.

L'action individuelle aspire donc à revêtir un caractère d'universalité, à se produire d'une façon qui puisse être comprise de tous. L'exemple de l'oeuvre d'art cherchait à rendre compte de ce caractère. Mais l'oeuvre esthétique est saisie affective avant d'être conceptualisée; cette richesse est en même temps une faiblesse et une limitation du fait même de son indétermination. C'est pourquoi l'oeuvre esthétique est insuffisante à elle seule pour permettre et expliquer une communication complète avec autrui.

Par sa précision et sa rigueur, le langage favorise davantage la transmission d'un message déterminé. Il est "l'équivalent maniable, animé, intellectualisé de l'univers entier» (p. 232). Le langage, en tant que moyen de communiquer avec l'univers et l'homme, est la synthèse des signes et des oeuvres de l'homme. Les mots, qui ne véhiculent pas tout le verbe intérieur et qui n'épuisent pas toute la pensée, une fois lâchés, dépassent toujours ce que tel homme pensait et ce qu'il voulait exprimer; ils expriment l'individuel et l'universel l'un dans l'autre. De plus, si toute action peut être exprimée et expliquée par la parole, c'est qu'elle est elle-même une parole implicite, un besoin de se révéler à tous; elle est un langage dans lequel les intelligences se rencontrent à cause de son caractère d'universalité que lui a injecté la pensée.

Blondel, qui ne fait pas ici oeuvre de métaphysicien, est bien conscient des difficultés « d'expliquer la transfusion des pensées et des motifs d'action d'une conscience fermée dans une conscience fermée» (p. 234). La pensée est la clé de voûte. Si l'oeuvre matérielle a dans le déterminisme des faits où elle prend place 
un caractère universel, si le langage est un signe de l'universel, c'est qu'ils sont tous deux liés à la pensée. En d'autres termes, l'action, édifiée en oeuvre, détachée de ses générateurs, vit, croit, et porte en elle cette étincelle d'une pensée qui cherche à se communiquer. Or, si toute pensée est personnelle, en ce sens qu'on ne peut dans ce cas se faire remplacer, elle est aussi une expression particulière porteuse d'universel : «là est le principe de la communion réelle des intelligences » (p. 234). Plus précisément, une pensée ne peut se dévoiler à elle-même et à autrui que par la médiation du signe. Tout signe est une médiation entre celui qui l'émet et celui qui le reçoit. Il a pour fonction d'exprimer une pensée, en désignant un objet.

Il serait intéressant et éclairant de consulter les développements sur le rôle du signe tels que présentés par Blondel dans La Pensée. Le signe est conçu comme la marque de l'homme sur le monde: «Pour créer un signe, pour concevoir un monde de notions et de représentations doublant en quelque sorte le monde réel, pour prétendre insérer dans l'univers donné un changement conscient et voulu, en un mot pour avoir l'initiative d'une idée et d'une intervention efficace, il faut que l'homme ait le sentiment immédiat et profond de sa supériorité sur les choses, de la transcendance de son pouvoir et de ses fins personnelles. Tandis que l'animal reste emprisonné et asservi dans l'ordre immanent des forces auxquelles il s'adapte comme une partie dans un tout, l'homme a constamment l'inquiétude, la nostalgie de ce qui n'est pas, de ce qui devrait être, de ce qu'il voudrait sentir ou produire; et c'est là le principe commun de l'art, de la science, de la moralité, de l'aspiration religieuse ${ }^{9}$.

C'est donc parce que toute action vraiment humaine s'imprègne de pensée qu'elle est une sorte d'idée vivante, qu'elle acquiert une valeur universelle, qu'elle est communicable et contagieuse. On pourrait dire inversement qu'il n'y a de pensée qu'incarnée et agie, et qu'une pensée agie est un exemple et un entraînement. Ainsi, ce que l'homme fait pour se connaître est fait pour être connu par d'autres. Le spectacle de sa raison devient le spectacle de la raison. Un acte voulu en est un orienté vers

9. La Pensée, t. 2, p. 111. 
ce qui peut comprendre et vouloir. On ne peut donc vivre pour soi ; tout sujet a des fenêtres ouvertes sur le dehors; on ne peut demeurer indifférent ou étranger aux suites de ses actes, de ses paroles et enseignements. L'action, édifiée en oeuvre, devient non seulement le produit mais aussi comme l'instrument et le lien d'une union plus réelle entre des consciences naturellement solitaires et inconnues les unes aux autres. Si les suggestions ressuscitent en d'autres consciences, c'est grâce à la double médiation du signe particulier et de l'idée générale dont l'union fait leur vie. Par ce qu'il y a de générique en tout acte humain, les hommes pénètrent tous les uns dans les autres.

De plus, l'action ou l'oeuvre constituée, qui dépasse toujours la prévision, contient plus que l'image peinte dans la conscience : «puisque l'action ajoute à la pensée et à l'intention un complément essentiel, puisque l'opération réalisée est une synthèse enrichie encore d'élétnents nouveaux, il est donc naturel que, sur d'autres consciences, l'acte revêtu de son accroissement sensible ait la double efficacité de ce qu'il a à la fois d'intelligible et de matériel » (p. 236). La nature profonde et durable de l'influence de l'action dépend donc de la manière dont en elle l'idée est unie à sa matière; plus ce lien est fort, plus la vie s'y incruste avec puissance et fécondité. Blondel propose comme exemple le cas du génie: "la destinée des grands inventeurs est ordinairement de trouver autre chose et plus qu'ils ne savent» (p. 236). L'oeuvre s'enrichit, sous cette poussée géniale, de ce que le temps y ajoute lentement, tout en demeurant elle-même. Parce que l'oetvre aide à découvrir des pensées et des idées qui, sans sa médiation, n'auraient pas effleuré au champ de la conscience claire, parce qu'elle est un acte, il est naturel qu'elle soit lourde de responsabilité pour quiconque enseigne ou agit : «le souci de la clarté et de la netteté est un souci moral» (p. 238). Dans ce qu'on fait et dit, il y a l'art de la vaccination intelllectuelle à pratiquer. Ce passage de Blondel condense bien ce qu'une analyse précédente a recueilli : "L'action est donc efficace et par ce qu'elle a de total et par ce qu'elle a de partiel, par ce qui est clair en elle et par ce qui est obscur, par son idée et par son corps, par la nécessité du déterminisme et par les suggestions de la vie spontanée, par l'originalité de son invention et par le principe universel qui rend toute idée accessible à tout esprit» (p. 238). 
Il est maintenant à se demander si cette transfusion des pensées et des oeuvres d'un individu en d'autres agents est encore le dessein du vouloir initial. En effet, l'action exercée sur des forces brutes n'a pas la même portée que celle exercée sur des consciences. Agir sur des esprits, soit par l'enseignement soit par l'exemple, c'est au fond prétendre retrouver chez eux son oeuvre ou un décalque de soi-même, c'est vouloir atteindre à une conformité la plus parfaite possible à son dessein : « Je suscite un autre moi $\gg$ (p. 240). D'ailleurs, n'est-ce pas ce à quoi visait l'action dès le début? Ainsi, l'homme exigerait de la part de ses semblables une passivité d'imitation et d'obéissance! Est-ce à ce prix qu'il doit satisfaire le besoin de se compléter? Mais s'il cherche en l'autre un complément de soi-même, n'est-ce pas plutôt parce qu'il suppose que cet autre peut le compléter, donc qu'il est lui aussi actif et capable d'initiative? Par conséquent, s'il est naturel de souhaiter que les autres se conforment à soi, il est aussi normal qu'ils conservent leur propre personnalité. On y voit apparaître ici une dialectique du commandement èt de l'obéissance, du besoin de se communiquer et du respect d'autrui, de la volonté de se retrouver en l'autre tout en le laissant être tel : «Nous voulons que les autres soient nous et nous voulons qu'ils restent eux 》 (p. 241).

A ces apparentes contradictions, Blondel répond qu'il s'agit là d'un seul et même désir: se répandre et s'accroître. Les contraires sont le prix offert et l'enjeu proposé à la volonté : «Chacun fait comme s'il agissait pour soi seul ; et chacun fait comme s'il agissait en l'autre et par l'autre» (p. 241). Le travail d'élaboration indispensable chez le destinataire pour retrouver l'esprit dans la lettre est la sauvegarde de la responsabilité des deux. Si une intervention apparaît à autrui comme un motif d'action qui n'enchaîne pas son choix, si on agit sur lui tout en le laissant agir en soi, c'est en raison de la portée universelle de l'action; la possibilité de l'influence, de même que la filiation et la transmission dans le monde des consciences, sont dues à ce caractère d'universalité. Certes, cette universalité n'est pas une garantie automatique du respect de l'intimité d'autrui; mais elle est comme un vaste terrain où peut se nouer une libre relation entre consciences. Et même si l'influence ne s'exerce pas de la même façon dans les divers esprits, il y a une greffe qui se fait par le 
caractère impersonnel de l'oeuvre et le caractère commun entre les différents esprits.

\section{III - CONCLUSION}

L'action est faite pour autrui : «Agir, c'est évoquer d'autres forces, c'est appeler d'autres moi » (p. 239). Voilà ce qu'a voulu montrer ce travail. Suivant le mouvement progressif et dialectique de la volonté initiale, dont l'ambition concrète est de tout s'égaler ou d'atteindre à l'unité, l'action est apparue comme l'exergie et l'allergie d'une synergie. Unissant en un faisceau les forces éparses de la vie, et manifestant la fonction médiatrice entre les énergies de l'activité corporelle et spirituelle, l'action a fait éclater la subjectivité en intersubjectivité. C'est ainsi qu'elle $\epsilon s t$ devenue «le ciment social » (p. 231), « la fonction sociale par excellence» (p. 239). Ce même mouvement volontaire, traversant les synthèses naturelles, biologiques, psychologiques, réfléchies, destinait l'action à la société. Il manifestait aussi les racines profondes de la personne sociale, et laisse prévoir comment la société, en plus d'être une oeuvre de raison et de liberté, est aussi une oeuvre ou une ébauche de la nature. On a vu que l'homme, non seulement a besoin d'autrui pour vivte, mais qu' $\mathrm{ll}$ a besoin de vivre pour autrui. Et les moyens de communication nécessaires lui permettant d'entrer en relation avec ses semblables, sont des signes expressifs : d'une part le langage verbal, d'autre part toute oeuvre humaine; ces signes, authentiques médiateurs de consciences, ont une portée universelle.

Il faudrait faire un pas de plus avec Blondel, à l'intérieur même de cet anneau social de la chaîne du déterminisme de l'action, et montrer comment l'action est faite pour être vécue avec autrui, dans une même communauté ; car une seule oeuvre de coopération ne satisfait pas complètement le vouloir. La nécessité d'une fusion plus intime parait, et c'est dans l'oeuvre de l'éducateur que se mesure le mieux la force et la faiblesse de l'action d'influence. Cette ardeur de prosélytisme, Blondel la rattache à la communication et à l'amour du vrai : "C'est tout un d'aimer la vérité et d'aimer les intelligences; de la connaître et de vouloir la répandre; de la chercher en soi et de la susciter en autrui; d'en vivre et d'en faire vivre les esprits» (p. 243). 
Mais la vérité n'est vivante que dans un esprit vivant: "c'est d'une personne qu'elle nous devient personnelle» (p. 244). Telle volonté exige d'autrui une union pleine, sans que l'unité et la dualité soient sacrifiées l'une à l'autre. Ce n'est plus d'actes convergeant en une oeuvre, ni de coaction ou d'influence qu'il s'agit, mais d'une union active et féconde. L'individu aspire à revivre en autrui. Désormais, le centre d'action de l'individu se transporte dans l'union intime avec une autre lui-même. L'action devient coénergie, échange de vie à vie. Ce secteur ouvre la voie à une analyse de la société proprement dite et aux trois niveaux de l'expansion sociale que sont la famille, la nation et l'humanité. Il mérite un examen sérieux que je me réserve de faire ailleurs.

En raison de son allure plus descriptive qu'explicative, $\epsilon t$ parce qu'il n'explore que l'Action de 1893 , on aura compris que cet exposé de la dimension sociale de l'action, retraçant la genèse de la vie sociale, n'appelle pas une appréciation de la pensée sociale de Blondel, ni de l'idée qu'il s'est faite de la société. Qu'il suffise de condenser dans ces quelques caractéristiques les résultats suivants.

1. Pour Blondel les phénomènes de société se trouvent inclus dans le programme général et dans l'exécution proprement dite de l'action. Sa philosophie les retrace et les explique à la manière d'une étape nécessaire et volontaire par laquelle l'homme passe pour se réaliser.

2. C'est ainsi qu'il se fait une loi de n'avancer que sous la pression d'une dialectique immanente et concrète. A chaque étape, il dégage un écart et une contradiction entre l'intention profonde de l'homme et l'objet limité qui est atteint, afin de mettre en évidence la volonté d'un perpétuel dépassement: l'autonomie ne s'acquiert que dans et par l'hétéronomie. Toujours, c'est le vouloir initial qui, concrètement c'est-à-dire en action, épuise tout, invente tout, admet tout pour se satisfaire; chaque objectif que l'homme s'assigne ne prend sa signification et sa valeur que par un vouloir infini.

3. Le progrès de l'aspiration volontaire l'amène à reconstruire, en le ratifiant, tout ce qui compose les milieux physique et psychologique, pour les prolonger dans le social: c'est ainsi 
que, dans son désir de rejoindre la volonté voulante, la volonté voulue, par la médiation de l'action, organise la vie individuelle (synergie), fait éclater la subjectivité (exergie), pour la lier à d'autres subjectivités (allergie), et veut pénétrer l'intimité même du collaborateur afin de réaliser une union active et féconde (coénergie).

4. La dimension sociale de l'homme plonge donc ses racines jusque dans le milieu cosmique, pour s'épanouir en pleine sphère spirituelle. L'action est à la fois substance de l'homme et génératrice de vie sociale ; l'homme est dans l'exacte mesure où il agit; être c'est agir, sans que pourtant aucun agir n'égale tout à fait l'être. L'homme se réalise dans les oeuvres qu'il produit; l'oeuvre opérée se détache d'une certaine manière de son auteur pour commencer une vie relativement indépendante et devenir à son tour féconde; elle façonne l'ouvrier et le milieu dans lequel elle se développe. L'action, oeuvre humaine, suscite la collaboration, révèle l'homme à lui-même et aux autres. L'action devient fonction sociale et s'épanouit en société. La société est une oeuvre de nature, de raison et de liberté ; son centre est dans l'individu; elle a un caractère intérieur.

5. La philosophie blondélienne de l'action est anti-individualiste, tout en refusant l'étiquette personnaliste; elle s'oppose à toute interprétation extrinséciste de la réalité sociale; elle se présente comme une philosophie de la solidarité. 